\title{
Clinical Study
}

Stereotactic

and Functional

Neurosurgery
Stereotact Funct Neurosurg 2015;93:206-211

DOI: $10.1159 / 000380827$
Received: November 14, 2014

Accepted after revision: February 10, 2015

Published online: April 21, 2015

\section{Deep Brain Stimulation Significantly Decreases Disability from Low Back Pain in Patients with Advanced Parkinson's Disease}

\author{
Heather Smith ${ }^{a}$ Lucy Gee ${ }^{a, c}$ Vignessh Kumar ${ }^{a}$ Adolfo Ramirez-Zamora ${ }^{b}$ \\ Jennifer Durphy ${ }^{b}$ Era Hanspal ${ }^{b}$ Anne Barba ${ }^{b}$ Eric Molho ${ }^{b}$ Damian Shin ${ }^{c}$ \\ Julie G. Pilitsis ${ }^{a, c}$ \\ Departments of a Neurosurgery and ${ }^{b}$ Neurology, and ${ }^{c}$ Center for Neuropharmacology and Neuroscience, Albany Medical \\ College, Albany, N.Y., USA
}

\section{Key Words \\ Deep brain stimulation - Low back pain · Parkinson's \\ disease $\cdot$ Subthalamic nucleus · Disability · Pain}

\begin{abstract}
Background: Up to $60 \%$ of Parkinson's disease (PD) patients suffer from low back pain (LBP) during the course of their disease. How LBP affects daily functional status and how to manage this aspect of PD has not been adequately explored. Methods: We examined 16 patients undergoing bilateral subthalamic nucleus deep brain stimulation (STN DBS) who met the inclusion criteria for moderate disability from LBP, as classified by the Oswestry Low Back Pain Disability Index (OLBPD). Results: Thirteen of 16 patients had attempted additional treatments for LBP, including medical management, massage, chiropractic, epidural steroid injections and/or surgery, with minimal relief. Following DBS, there was a significant improvement in the OLBPD at both the 6-month and 1 -year time points ( $p<0.02, p<0.005$, respectively). A mean improvement of $31.7 \%$ on the OLBPD score was noted. The Visual Analogue Scale (VAS) similarly decreased significantly at 1 year $(p=0.015)$. There was no correlation between the OLBPD score and other measures, including the Unified Parkinson's Disease Rating Scale (UPDRS), age and other non-
\end{abstract}

motor symptoms. Conclusion: Given the prevalent yet undertreated disability associated with LBP in PD, these results are novel in that they show that STN DBS has a significant positive effect on disability associated with LBP.

(C) 2015 S. Karger AG, Base

\section{Introduction}

Pain may be a presenting symptom of Parkinson's disease (PD), and it is a common complaint in PD patients as the disease advances. The etiology and classification of pain in PD is not well understood, and in many cases pain is mistakenly attributed to comorbid conditions rather than being a sequela of the disease itself [1]. In order to reduce the severity of this pain, it is not uncommon for patients to undergo multiple medical treatments as well as surgery for pain relief. In fact, up to $60 \%$ of PD patients have pain complaints over their lifetime as compared to $25-50 \%$ of older adults without PD $[2,3]$. The McGill pain questionnaire is a clinical tool commonly used to evaluate the frequency, duration, character, severity, location and temporal qualities of pain. Goetz et al. [4] used this tool to determine whether pain was related to PD or other causes in $95 \mathrm{PD}$ patients. Patients reported that $46 \%$

\section{KARGER 125}

(c) 2015 S. Karger AG, Base

1011-6125/15/0933-0206\$39.50/0

E-Mail karger@karger.com

www.karger.com/sfn
Julie G. Pilitsis, MD, $\mathrm{PhD}$

AMC Neurosurgery Group

47 New Scotland Avenue, MC 10

Albany, NY 12208 (USA)

E-Mail jpilitsis@yahoo.com 
of the pain was directly related to their symptoms of PD. Further, patients with PD for $>5$ years report a 35\% higher incidence of pain as compared to patients with early stages of PD [5].

The most common pain complaint in PD is low back pain (LBP), affecting $28 \%$ of patients who have pain [1]. Patients may describe this LBP to be: musculoskeletal (pain of the joints and muscles), radicular (pain caused by nerve root irritation), dystonic (pain caused by a severe muscle spasm following a twisting movement of a muscle), akathisic (an uncomfortable sensation accompanied by restlessness) or central (stabbing, burning pain as a direct result of dopamine insufficiency) [6,7]. Depending on the type of LBP, treatments may include medication, physical therapy, epidural steroid injections, chiropractic and/or acupuncture. Studies have reported that between $34 \%$ and $58 \%$ of patients with LBP take some form of analgesic medication for pain relief $[8,9]$. Rarely, surgery may be required [2].

Recently, it has been noted in a number of case series that deep brain stimulation (DBS) may alleviate pain in PD patients. Specifically, unilateral and bilateral DBS of the globus pallidus was shown to improve pain by $80 \%$ and limb dystonia by $90 \%$ [10]. Bilateral subthalamic nucleus stimulation (STN DBS) was shown to improve pain in $50 \%$ of PD patients after a period of 5 years, particularly when related to dystonia [11]. A recent report showed in a prospective case study that pain improved in PD patients undergoing bilateral STN DBS [1]. Multiple outcome measures were used including change in pain prevalence following surgery, changes in motor function as well as characteristics of pain and other nonmotor symptoms. It was found that pain intensity and nonmotor symptoms significantly improved following STN DBS and that dystonic and musculoskeletal pain responded well to STN DBS. Additionally, strong correlations were found between changes in pain intensity and overall quality of life [1]. While Cury et al. [1] state that $28 \%$ of their patients had primarily LBP, the effects of STN DBS on LBP alone and the disability it causes have not been further examined. Here, we evaluate the impact of STN DBS on functional disability from LBP, using the Oswestry Low Back Disability Index (OLBPD).

\section{Methods}

\section{Participants}

All subjects in this study were patients undergoing bilateral STN DBS treatment for PD at the Albany Medical Center and were consecutively enrolled. Those who qualified for surgical treatment completed the Unified Parkinson's Disease Rating Scale (UPDRS) and neuropsychological testing as part of the routine preoperative workup. Patients who did not improve more than $30 \%$ on CAPSIT on/off medication testing were not considered acceptable candidates, as well as those who demonstrated dementia or significant cognitive impairment at baseline testing. Subjects who could not complete testing due to language barriers and/or dementia were excluded from the study. Subjects included in the study fell in the categories of 'moderate disability,' 'severe disability' or 'crippling back pain' as defined by the OLBPD. Institutional review board approval for the study was obtained.

\section{Lower Back Pain and Disability Assessment}

After giving their informed consent, participants completed the OLBPD, which was given within 1 month preoperatively and at the 6-month and 1-year follow-up appointments to track changes in pain. The OLBPD has 10 categories (pain intensity, personal care, lifting, walking, sitting, standing, sleeping, sex life, social life and traveling), and patients are scored from 0 to $100 \%$ into categories of minimal disability (0-20\%), moderate disability (21-40\%), severe disability (41-60\%), crippling back pain (61-80\%) and bedbound (81-100\%). Questions evaluate how the patient had been feeling over a period of time prior to answering the questionnaire with their medication and stimulator (when applicable) on. Participants were also asked preoperatively and 1 year postoperatively to rate their global pain on the Visual Analogue Scale (VAS) of $0-10$ while on medication. These scores represent how the patient was feeling right at that moment. Autonomic dysfunction was assessed at the same time points using the Scales for Outcomes in Parkinson's Disease-Autonomic (SCOPA-AUT), and sleep dysfunction was assessed using the Parkinson's Disease Sleep Scale (PDSS). Depression and anxiety were assessed preoperatively and 1 year postoperatively in the patients' standard neuropsychological evaluation using Beck's Depression Inventory and the StateTrait Anxiety Inventory, respectively. All clinical assessments were completed with a research associate present to assist with writing, as this is often difficult in this patient population off medication. The research assistant acted as a scribe and did not influence the answers given by the patients.

\section{Data Analysis}

All data are expressed as mean \pm SEM and were analyzed for significance using either a paired-sample t test or Pearson's correlation analyses in SPSS (IBM SPSS Statistics for Windows, version 22.0, Armonk, N.Y.: IBM Corp.). For all statistical tests, a value of $\mathrm{p}<0.05$ was considered significant.

\section{Results}

\section{Demographics}

The participants underwent surgery at a mean age of $59.4 \pm 1.7$ years with a mean disease duration of $12.2 \pm$ 1.0 years. The mean age of disease onset in the cohort was $47.1 \pm 2.0$ years. Ten males and 6 females participated in our study. Further demographic information can be appreciated in table 1 . 


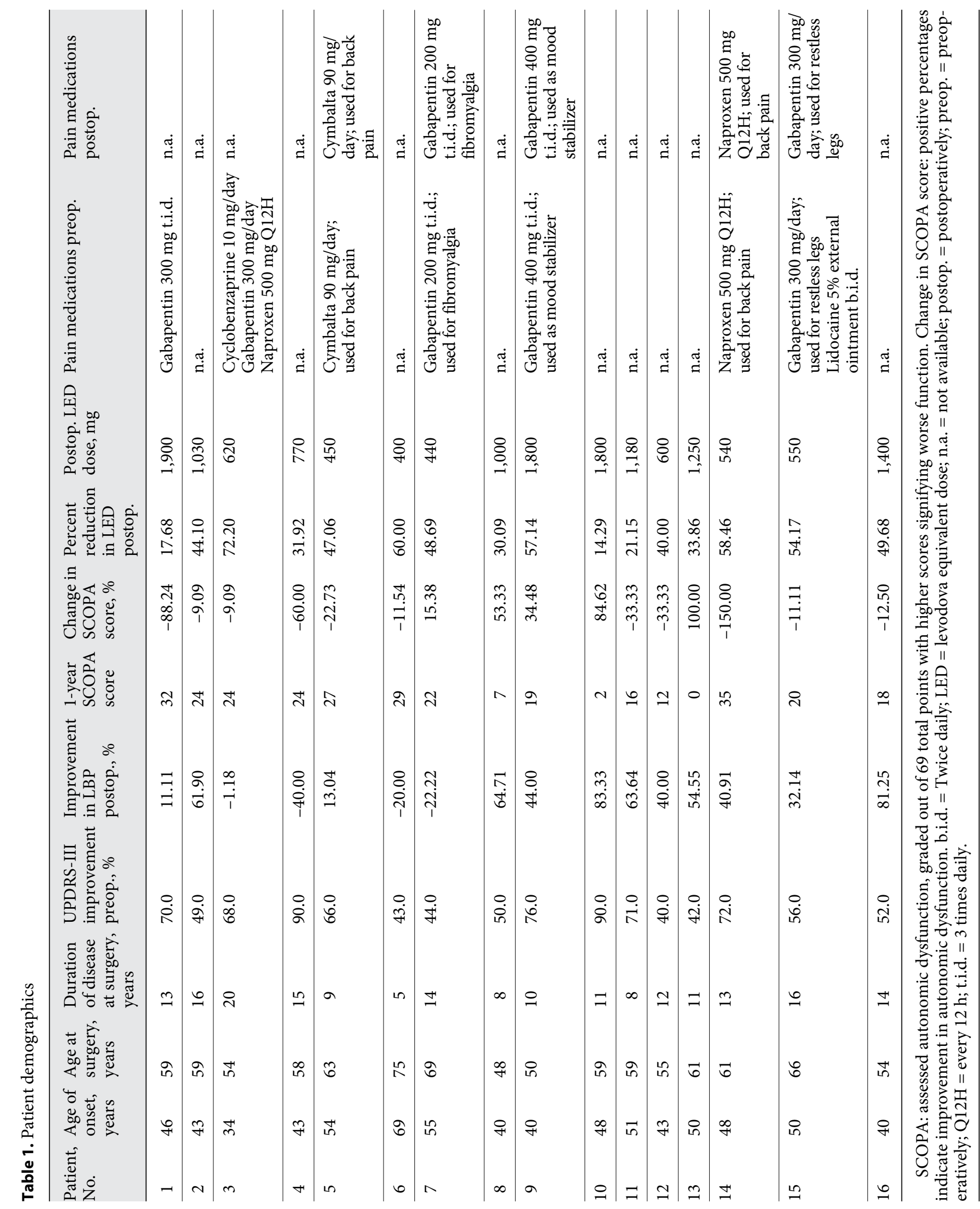




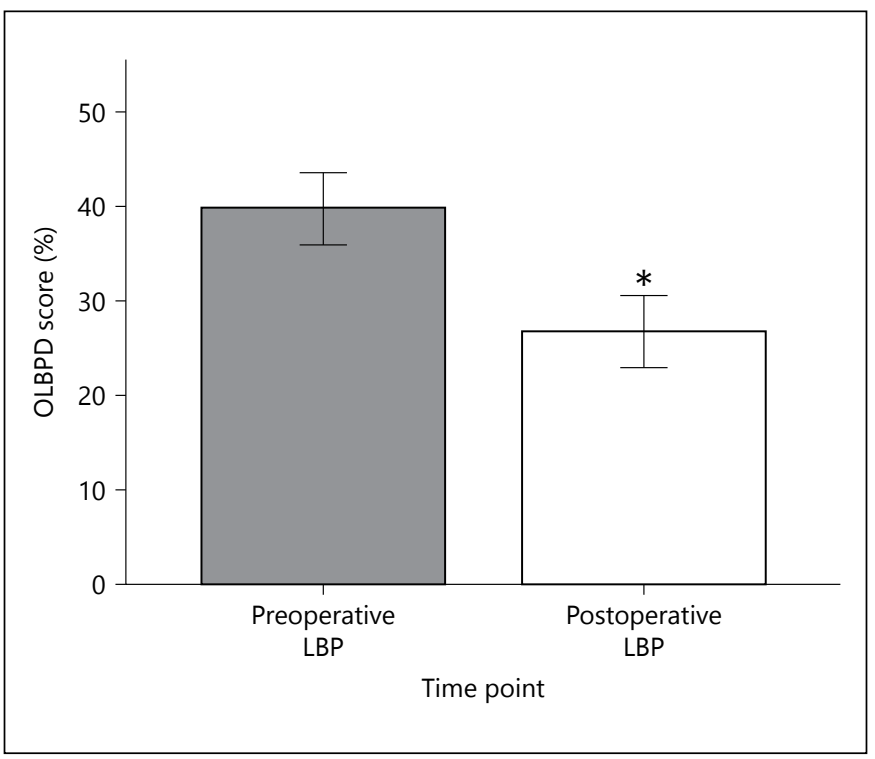

Fig. 1. Significant reduction in LBP following bilateral STN DBS. $* \mathrm{p}=0.002$.

\section{Low Back Pain}

Analysis of the OLBPD completed by the 16 participants with more than moderate LBP indicated a significant decrease in back pain at both the 6-month follow-up $[\mathrm{t}(15)=2.666, \mathrm{p}=0.017]$ and the 1 -year follow-up [ $\mathrm{t}(15)=$ $3.624, \mathrm{p}=0.002]$ in comparison to preoperative scores (fig. 1). This finding corresponded with the significant decrease in global pain, as measured with VAS preoperatively and 1 year postoperatively $[\mathrm{t}(15)=2.739, \mathrm{p}=0.015$ ] (fig. 2). Global pain scores remained at a level of 0 at all continued follow-up appointments, which ranged from 13 to 33 months after surgery. Similarly, the motor evaluation of the UPDRS (part III) significantly improved in the group, thus indicating a decrease in motor disability following surgery $[t(14)=5.723, p=0.000053$; mean improvement of $51.5 \%]$.

Preceding surgery, 7 patients were on pain medications. Six of 7 patients were taking medications for their back. Two of those 6 patients remained on medications (Cymbalta and Naprosyn). The other 4 patients were weaned off their medications. The medications for the other patient and for those patients that were on multiple medications were prescribed for reasons other than back pain (e.g. restless legs, fibromyalgia, depression). Four of 16 patients were undergoing alternative treatment for LBP (physical therapy, epidural steroid injections, chiropractic therapy), all of whom were taking noncontrolled substances to also medically manage

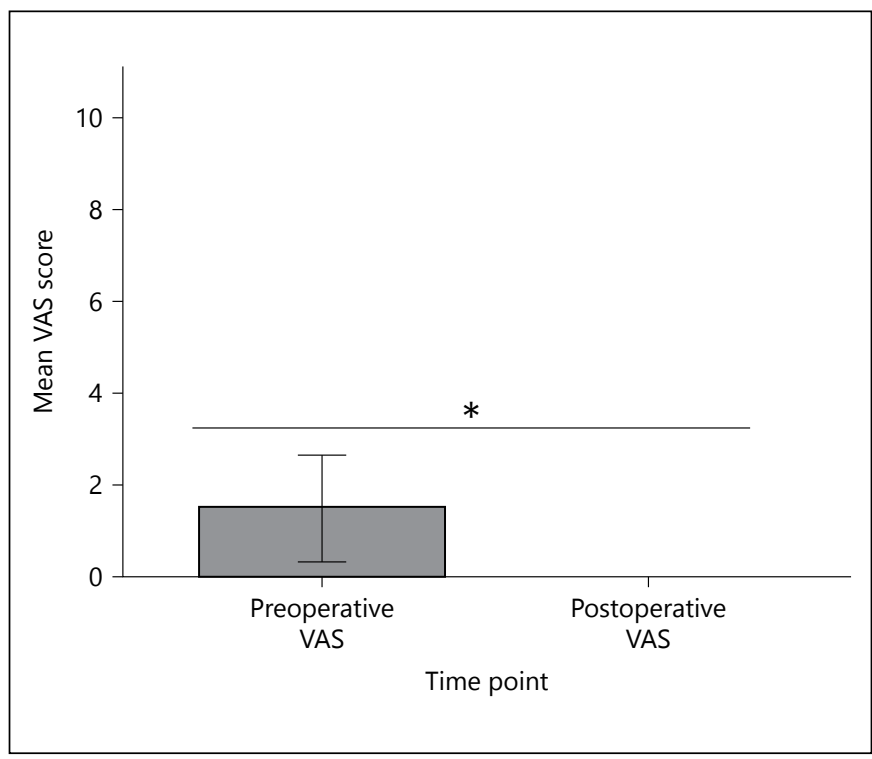

Fig. 2. Significant decrease in global pain postoperatively as measured by the VAS. ${ }^{*} \mathrm{p}=0.015$.

their pain. None of the 4 patients continued the alternative therapies postoperatively. Improvements in LBP did not correlate with preoperative usage of alternative therapies, UPDRS motor score, age, daily levodopa equivalent dose or improvement in sleep. Autonomic dysfunction, as measured with the SCOPA-AUT, did not improve postoperatively $(\mathrm{p}>0.5)$ and did not correlate with improvement in disability from LBP. Oneyear neuropsychological data were available for 13 of 16 patients, and the OLBPD score showed no correlation with depression or anxiety.

\section{Discussion}

Our study is the first to show that STN DBS reduces functional disability due to LBP in PD. Further, the VAS scores in our patient cohort parallel the mounting evidence that STN DBS can reduce pain in PD patients [5]. These results are exciting as they support positive improvements for patients after STN DBS.

Until recently, nonmotor symptoms of PD have been underappreciated and remained undertreated. Disability associated with LBP has been described as the most common cause of activity limitation in adults [12]. According to a cross-sectional study in 2006, the point prevalence of LBP in PD patients $(60 \%)$ was significantly higher than that in age-matched control patients 
with cardiovascular illness or diabetes (23\%) as well as the general elderly population (32\%) [13]. Further, a significantly larger percentage of PD patients (95.2\%) report that their back pain is chronic ( $>12$ months) when compared with controls (73.9\%). We predicted that in our patient cohort those patients with autonomic dysfunction might have a higher incidence of LBP associated with increased falling and postural instability [14]; however, this was not the case. Some authors suggest that the high prevalence of chronic LBP in PD may be caused by alterations in posture during the course of the disease or may be due to differences in muscle dynamics, causing joint trauma and increasing pain prevalence [13]. Finally, some evidence suggests that the basal ganglia are involved in sensory processing, and thus PD may result in aberrant nociceptive processing [15].

Chronic pain in PD has become a major focus of clinical research as motor symptoms are treated more efficiently with dopaminergic medication and DBS. It is reported as the third most bothersome symptom of PD, second only to tremors/shaking and lack of mobility [16]. In some patients, pain even overshadows the motor symptoms of PD [17]. Currently, depending on presentation, PD-related pain is treated with NSAIDs, opioid analgesics or antiparkinsonism therapies [6]; however, as with the motor symptoms, these treatments become less effective as patients reach a more advanced stage of disease. STN DBS has also been shown to alter sensory pain thresholds and reduce pain in PD patients in a number of studies [18-21]. In comparison to pain treated with levodopa, STN DBS provides a superior analgesic response $[15,22]$.

The mechanism by which STN DBS modulates neural activity in PD remains unclear. Previous research suggests that high-frequency stimulation of the subthalamic nucleus causes an inhibition of local neurons, which reduces the inhibitory output of the globus pallidus and the signal-to-noise ratio on the thalamus and allows movement. Others suggest that multiple mechanisms may be in play, including a depolarization block or activation of passing efferent or afferent axons [23]. Animal studies demonstrated that nociceptive inputs from the cortex, thalamus and amygdala were processed in the striatum and globus pallidus and that the activation of the dopamine receptor subtype $\mathrm{D}_{2}$ in the striatum could reduce pain [15]. Overall, this evidence suggests that STN DBS may reduce LBP by modulating neurons in the basal ganglia governing sensory processing.
Our results support others who have found that STN DBS reduces pain in PD patients. In our patient cohort, the OLBPD indicated that 9 patients had moderate disability (21-40\%), 6 had severe disability (41-60\%) and 1 had crippling back pain (61-80\%). One year following the STN DBS, 7 patients had minimal disability $(0-20 \%), 6$ had moderate disability and 3 had severe disability. Two patients had increases in the OLBPD scores at 6 months; however, they were decreased again at 1 year. The number of patients taking medications to treat their pain was reduced. No patients scored between 61 and 100\% (crippling disability) at 1 year, and all reported a VAS score of 0 . Interestingly, while the average preoperative VAS scores were much higher, some patients scoring highly on the OLBPD scale also reported a VAS score of 0 . This finding seems contradictory; however, it could be due to the patients' perception of physical pain. Improvement in sleep, depression and anxiety did not correlate with the OLBPD improvements, and we do not think those factors played a role in altering that perception. Significant improvements in UPDRS motor scores did correlate with decreases in the OLBPD; however, only a few of the questions pertained directly to activities involving significant amounts of movement; thus, the improvement in disability scores may have occurred through another pathway after STN DBS surgery. We believe that all STN DBS candidates could benefit, as improvements in LBP disability did not correlate with the UPDRS motor score, age or levodopa equivalent dose. Limitations of our study include the small sample size, a potential bias in patient selfreporting and a potential placebo effect.

In conclusion, our results are the first to show a reduction in disability measured by the OLBPD after STN DBS and support similar reports showing that STN DBS improves pain in PD patients. Future studies will be necessary to address the mechanisms by which this phenomenon occurs.

\section{Disclosure Statement}

Ms. Lucy Gee received funding from NIH grant 5 T35 HL 071483. Dr. Julie G. Pilitsis is a consultant for Medtronic, St. Jude Medical and Boston Scientific and receives grant support from Medtronic, Boston Scientific, St. Jude Medical and NIH 1R01CA166379. Dr. Adolfo Ramirez-Zamora is a consultant for Teva Neuroscience and received clinical trial support from Boston Scientific. Dr. Eric Molho is a consultant for Lundbeck, US WorldMeds and Merz and has received speaking honoraria from US WorldMeds and receives grant support from the Cure Huntington Disease Initiative, Kyowa, Teva, US WorldMeds, Aspen, Acadia, Merz and Boston Scientific. 


\section{References}

1 Cury RG, Galhardoni R, Fonoff ET, dos Santos Ghilardi MG, Fonoff F, Arnaut D, Myczkowski M, Marcolin MA, Bor-Seng-Shu E, Barbosa ER, Teixeira MJ, de Andrade DC: Effects of deep brain stimulation on pain and other nonmotor symptoms in Parkinson disease. Neurology 2014;83:1-7.

2 Beiske AG, Hoge JH, Ronningen A, Svensson E: Pain in Parkinson's disease: prevalence and characteristics. Pain 2009;141:173-177.

3 Silva EG, Viana MA, Quagliato EM: Pain in Parkinson's disease: analysis of 50 cases in a clinic of movement disorders. Arq NeuroPsiquiat 2008;66:26-29.

4 Goetz CG, Tanner CM, Levy M, Wilson R, Garron DC: Pain in idiopathic Parkinson's disease. Movement Disord 1986;1:45-49.

5 Kim HJ, Paek SH, Kim JY, Lee JY, Lim YH, Kim MR, Kim DG, Jeon BS: Chronic subthalamic deep brain stimulation improves pain in Parkinson disease. J Neurol 2009;255: 1889-1894.

6 Ford B: Pain in Parkinson's disease. Movement Disord 2010;25:98-103.

7 Hanagasi HA, Akat S, Gurvit H, Yazici J, Emre M: Pain is common in Parkinson's disease. Clin Neurol Neurosur 2010;113:1113.

$\checkmark 8$ Lee MA, Walker RW, Hildreth TJ, Prentice WM: A survey of pain in idiopathic Parkinson's disease. J Pain Symptom Manag 2006; 32:462-469.

9 Broetz D, Eichner M, Gasser T, Weller M, Steinbach JP: Radicular and nonradicular back pain in Parkinson's disease: a controlled study. Movement Disord 2007;22: 853-856.
10 Loher TJ, Burgunder JM, Weber S, Sommerhalder R, Krauss JK: Effect of chronic pallidal deep brain stimulation on off period dystonia and sensory symptoms in advanced Parkinson's disease. J Neurol Neurosur Ps 2002;73: 395-399.

11 Krack P, Batir A, Van Blercom N, Charbades S, Fraix V, Ardouin C, Koudsie A, Limousin PD, Benazzouz A, LeBas JF, Benabid A, Pollak P: Five-year follow-up of bilateral stimulation of the subthalamic nucleus in advanced Parkinson's disease. New Engl J Med 2003;349: 1925-1934.

$\checkmark 12$ Loney PL, Stratford PW: The prevalence of low back pain in adults: a methodological review of the literature. Phys Ther 1999;79:384396.

13 Etchepare F, Rozenberg S, Mirault T, Bonnet AM, Lecorre C, Agid Y, Bourgeois P, Fautrel B: Back problems in Parkinson's disease: an underestimated problem. Joint Bone Spine 2006;73:298-302.

$14 \mathrm{Ha} \mathrm{AD}$, Brown CH, York MK, Jankovic J: The prevalence of symptomatic orthostatic hypertension in patients with Parkinson's disease and atypical parkinsonism. Parkinsonism Relat Disord 2011;17:625-628.

15 Conte A, Khan N, Defazio G, Rothwell JC, Berardelli A: Pathophysiology of somatosensory abnormalities in Parkinson's disease. Nat Rev Neurol 2013;9:687-697.

16 Uebelacker LA, Epstein-Lubow G, Lewis T, Broughton MK, Friedman JH: A survey of Parkinson's disease patients: most bothersome symptoms and coping preferences. J Parkinsons Dis 2014;4:717-723.
17 Quittenbaum BH, Grahn B: Quality of life and pain in Parkinson's disease: a controlled cross-sectional study. Parkinsonism Relat Disord 2004;10:129-136.

18 Ciampi de Andrade D, Lefaucheur JP, Galhardoni R, Ferreira KS, Brandão Paiva AR, Bor-Seng-Shu E, Alvarenga L, Myczkowski ML, Marcolin MA, de Siqueira SR, Fonoff E, Barbosa ER, Teixeira MJ: Subthalamic deep brain stimulation modulates small fiber-dependent sensory thresholds in Parkinson's disease. Pain 2012;153:1107-1113.

19 Gierthmuhlen J, Arning P, Binder A, Herzog J, Duschl G, Wasner G, Baron R: Influence of deep brain stimulation and levodopa on sensory signs in Parkinson's disease. Movement Disord 2010;25:1195-1202.

20 Spielberger S, Wolf E, Kress M, Seppi K, Poewe W: The influence of deep brain stimulation on pain perception in Parkinson's disease. Movement Disord 2011;26:1367-1368.

21 Maruo T, Saitoh Y, Hosomi K, Kishima H, Shimokawa T, Hirata M, Goto T, Morris S, Harada Y, Yanagisawa T, Aly MM, Yoshimine T: Deep brain stimulation of the subthalamic nucleus improves temperature sensation in patients with Parkinson's disease. Pain 2011;152:860-865.

22 Surucu O, Baumann-Vogel H, Uhl M, Imbach LL, Baumann CR: Subthalamic deep brain simulation versus best medical therapy for L-dopa responsive pain in Parkinson's disease. Pain 2013;154:1477-1479.

23 Rosa M, Giannicola G, Marceglia S, Fumagalli M, Barbieri S, Priori A: Neurophysiology of deep brain stimulation. Int Rev Neurobiol 2012;107:23-55. 